\title{
Mural endocarditis - by default
}

\section{Anupa Patel, Dominic Kakooza, Thomas Kalk and Nqoba Tsabedze}

Division of Cardiology, Department of Internal Medicine, School of Clinical Medicine, Faculty of Health Sciences, University of the Witwatersrand and Charlotte Maxeke Johannesburg Academic Hospital, Parktown, Johannesburg, South Africa

Address for correspondence:

Dr Anupa Patel

5 Jubilee Road

Parktown

Johannesburg

2193

South Africa

Email:

dranupa.patel@gmail.com

\section{INTRODUCTION}

Infective endocarditis is typically associated with valvular structures, in particular valvular insufficiency. The pathogenesis thereof is related to the high velocity non-laminar jet which causes endothelial disruption and platelet fibrin deposition, resulting in thrombus generation susceptible to bacterial infection. (1) Non-valvular mural endocarditis similarly occurs where there is high non-laminar blood flow which empties into a low pressure structure such as in cardiac structural abnormalities (e.g., restrictive ventricular septal defects, patent ductus arteriosus, arteriovenous fistulae or connections and/or where there may be material susceptible to infective processes such as mural thrombi, cardiac tumours, or prosthetic materials. ${ }^{(2)}$ About $70 \%$ of patients with mural endocarditis have a predisposing risk factor, including a immunocompromised state, intravenous drug use or recent surgery and these patients tend to culture virulent organisms such as Staphylococcus Aureus. ${ }^{(3)}$ Guidelines for managing infective endocarditis recommends urgent surgery for patients with large vegetations, embolic phenomena and major valvular complications. ${ }^{(4)}$

\section{CASE REPORT}

A 33-year-old woman presented with transient decreased vision in her left eye associated with persistent headaches. She

\section{ABSTRACT}

We report on a case of a 33-year-old female patient with known adult congenital heart disease in the form of a coronary cameral fistula who, on prior presentation, had refused treatment and subsequently defaulted follow-up, but unfortunately developed a serious and rare complication. She presented with non-specific neurological symptoms of transient visual loss and headaches. Clinical examination revealed aortic regurgitation with peripheral features of infective endocarditis. This was confirmed with transthoracic echocardiogram, but additionally, we found mural endocarditis of the right atrial posterior wall. An initial CT brain scan and ophthalmology assessments were negative, blood cultures were positive and confirmed infective endocarditis, and she was referred for urgent cardiothoracic surgery. Unfortunately, she had further complications with a large intracranial haemorrhage which ultimately led to her death. This case illustrates a rare entity with disastrous complications. SAHeart 2021;18:64-67

was known to have been diagnosed previously with a coronary cameral fistulae (CCF) extending from her left coronary cusp into the right atrium (RA), but despite advice had refused surgery at the time and furthermore, she defaulted on her follow-up. On presentation to our casualty department, she was haemodynamically stable, not in heart failure but was pyrexial with peripheral features of infective endocarditis. Additionally, she had a wide pulse pressure (BP 125/48) and a bounding pulse with a positive Durozies' sign. Auscultation revealed a continuous murmur (right parasternal border) and long, early diastolic murmur (left second Intercostal space). Transthoracic echocardiography demonstrated vegetations of the aortic valve (AV) complicated by severe aortic regurgitation (Video I and 2). The left ventricle was dilated and left and right ventricular function was preserved. Large oscillating masses were found adherent to the posterior (RA) wall. (Figure I, Figure 2 and Video 3). Colour Doppler imaging demonstrated high flow through these mural masses. (Figure 3 and Video 4). The dilated, anomalous coronary artery, measuring $14 \mathrm{~mm}$, was seen emptying into the RA and the turbulent flow from the 


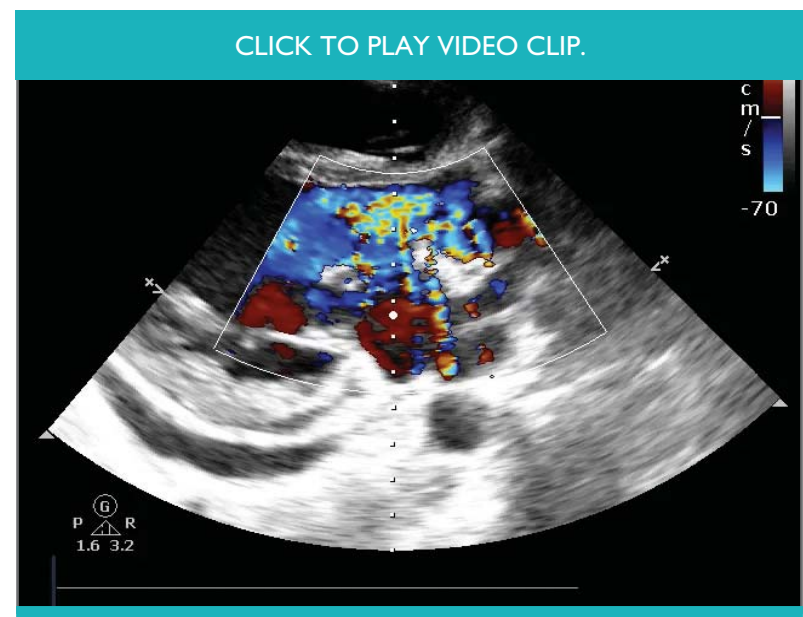

VIDEO I: PLAX view with colour doppler imaging demonstrating aortic regurgitation.

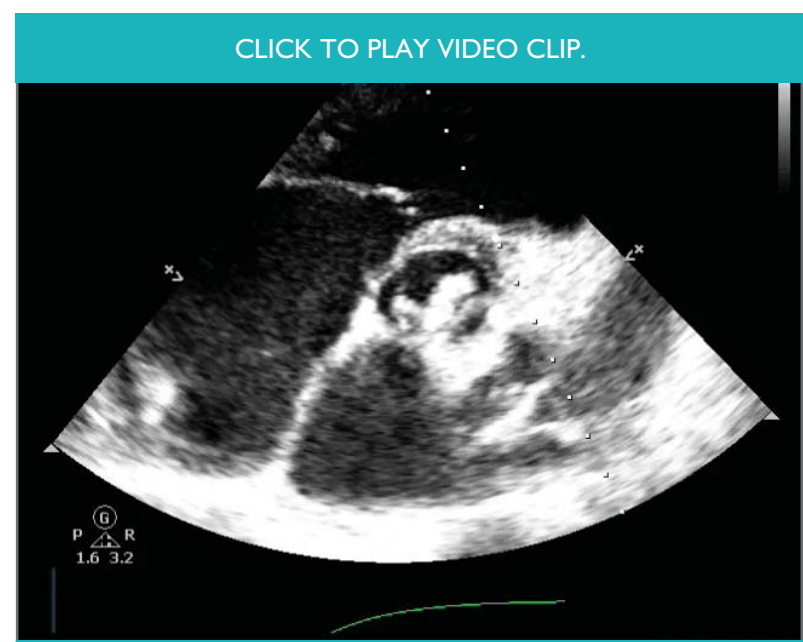

VIDEO 2: Oblique SAX view at level of LVOT demonstrating AV vegetations prolapsing into LVOT.

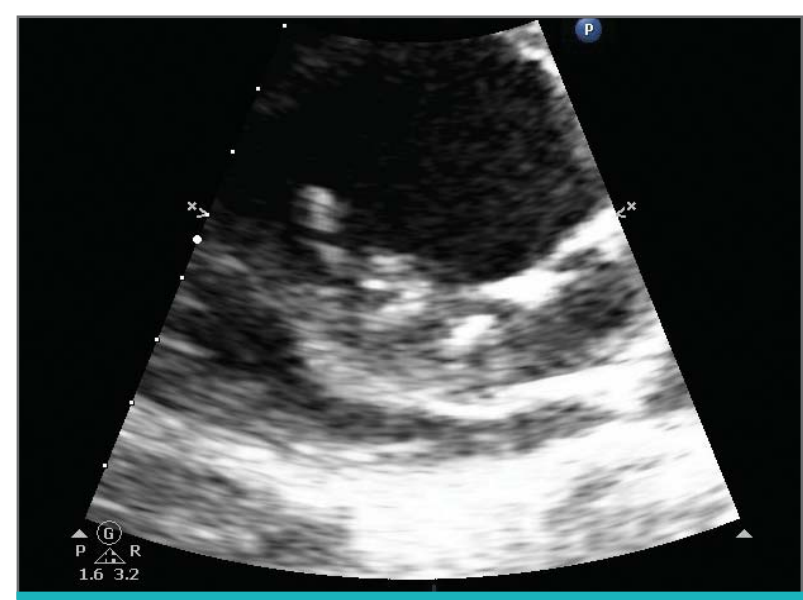

FIGURE I: Still image of Focused RV A4Ch view with masses seen adherent to RA wall.

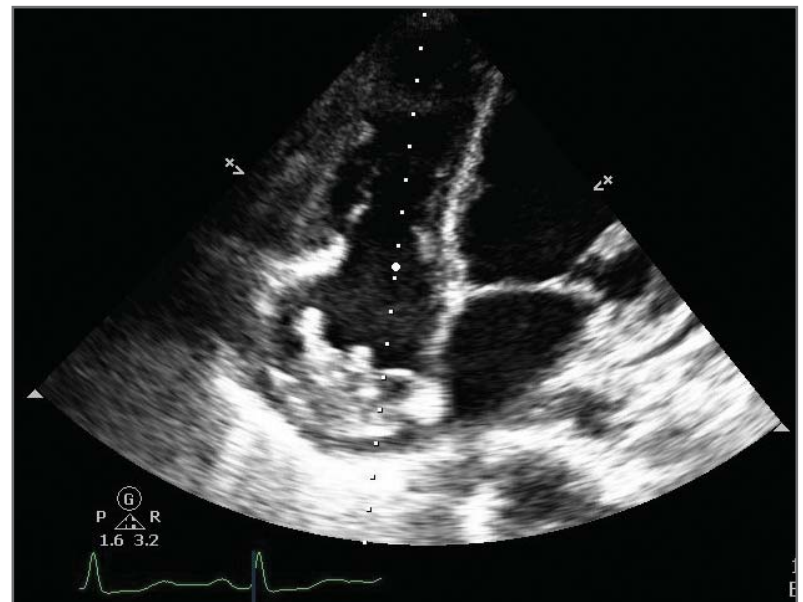

FIGURE 2: Still image in oblique SAX at AV level with zoomed view of RA wall.

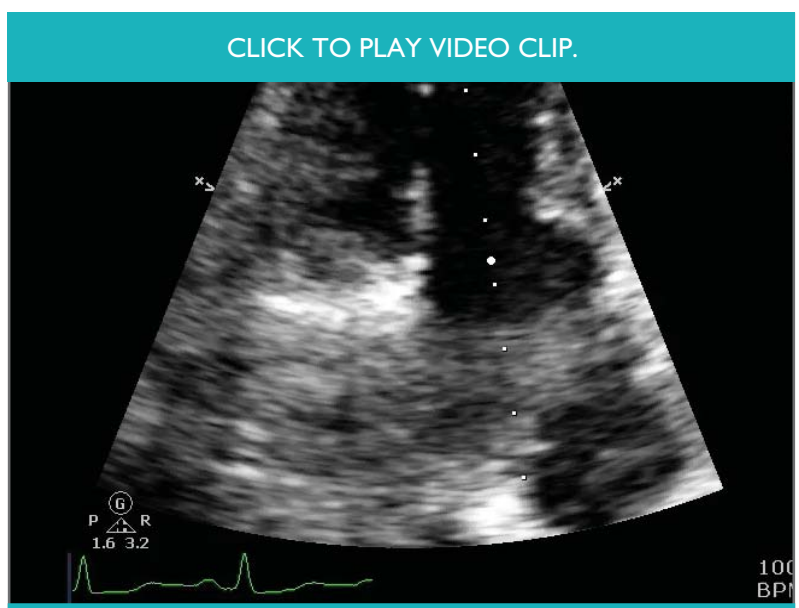

VIDEO 3: Zoomed view of RA demonstrating oscillating masses adherent to the RA posterior wall in Focused RV A4Ch view.

exit of this CCF was directed towards the posterior wall of the RA and was seen swirling through and around these masses. (Figure 4 and Video 5). Two sets of blood cultures confirmed Streptococcus Viridans species. Initial CT Brain (CTB) ruled out embolic complications. Ophthalmology assessment did not reveal any abnormalities. The previous cardiac catheterisation was reviewed, this confirmed a large aneurysmal left main artery, with an anomalous course draining into the RA. Proximally it gave rise to normal left anterior descending and circumflex coronary arteries. The right coronary artery was normal. The large CCF was confirmed at that time with a significant shunt (calculated Qp:Qs of 1,84:1). It was decided not to repeat the cardiac catheterisation on this presentation due to 


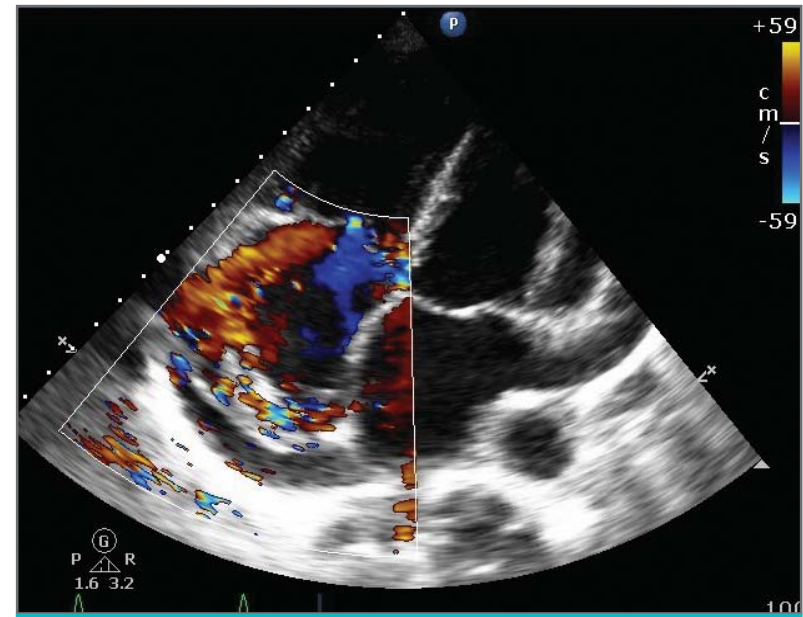

FIGURE 3: Still image of Focused RV A4Ch view with Colour doppler imaging focussed on RA demonstrating turbulent colour flow around the masses attached to RA wall.

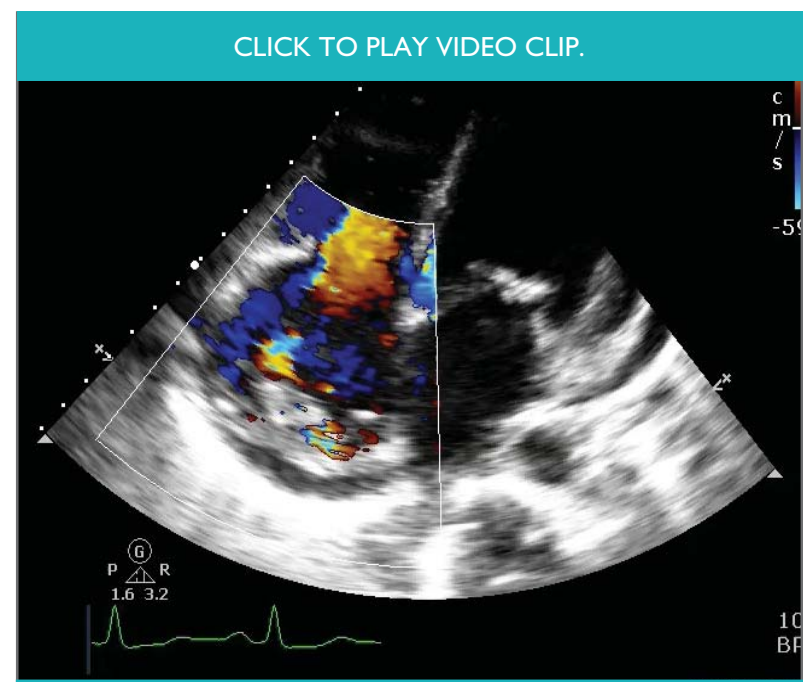

VIDEO 4: Focused RV A4Ch view with Colour Doppler imaging demonstrating turbulent flow around the oscillating masses adherent to the RA wall.

the risk of embolisation from the $\mathrm{AV}$ vegetations. The patient was referred for urgent cardiothoracic surgery. Unfortunately, preoperatively, she deteriorated suddenly and sustained a cardiorespiratory arrest. Initial cardiorespiratory resuscitation was successful and a repeat CTB revealed a large intracranial haemorrhage. Surgery was cancelled pending neurosurgical management but unfortunately her neurological status worsened and despite neurosurgical management, she subsequently died a few days later.

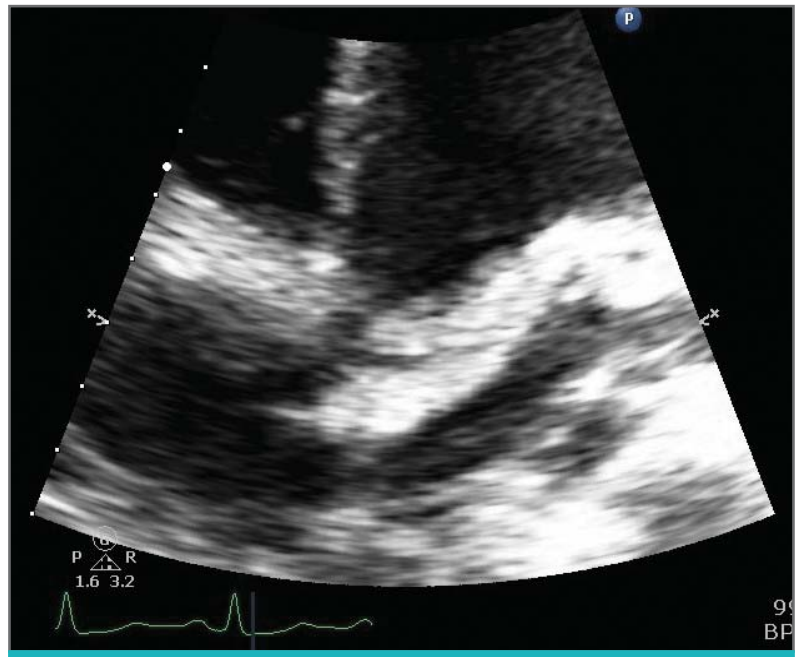

FIGURE 4: Still image in in Oblique A4Ch view with zoomed view of dilated anomalous coronary artery entering $\mathbf{R A}$.

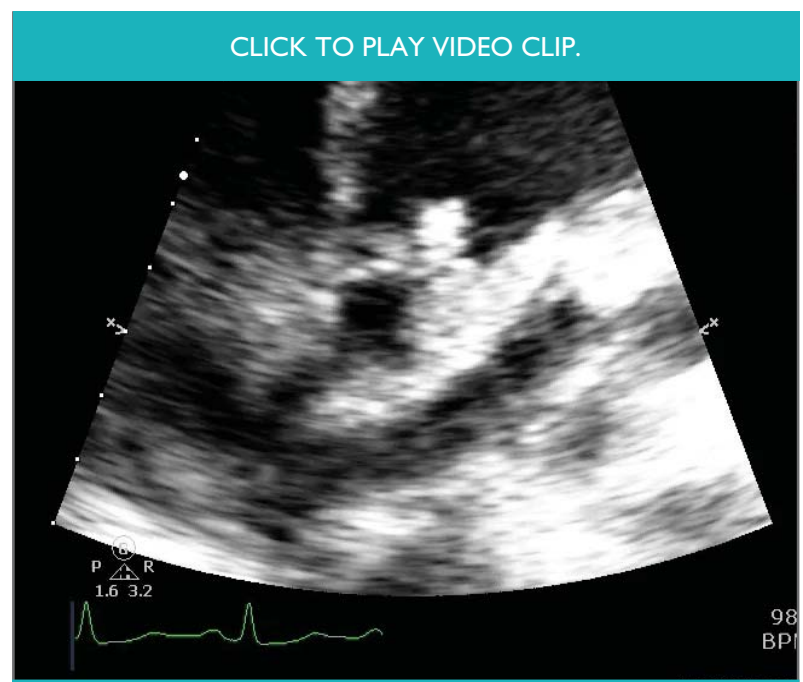

VIDEO 5: Zoomed view of dilated anomalous coronary artery entering RA in Oblique A4Ch view.

\section{DISCUSSION}

Diagnosing mural endocarditis can be challenging. Echocardiography is often the primary diagnostic modality used. The characteristics of a mural mass such as location, mobility, attachment and appearance can give important clues to the aetiology of the mass. ${ }^{(5)}$ In our patient, the size and location of the multiple mural masses together with high flow seen along the posterior RA wall made a diagnosis of thrombus (thrombosis) unlikely. The lack of echocardiographic features such as: 
infiltration of the myocardium, crossing of anatomical planes and pericardial effusion was against a diagnosis of a cardiac malignancy. The CCF was seen emptying into the RA with blood flow directed to the posterior wall and the AV had obvious oscillating masses. The clinical picture confirmed IE. These mural masses were thought to be infected mural endocarditis, seeded from the vegetations of the AV via the shunted blood from the CCF. Appropriate and early management may lead to good outcomes in the management of these patients. Unfortunately, our patient presented late with severe sequelae and subsequently experienced further complications.

This case demonstrates that using a simple approach encompassing clinical information and good echocardiography imaging may aid diagnosis. Furthermore, it demonstrates the poor outcomes that may occur in delayed presentations with consequent delayed management.

\section{Conflict of interest: none declared.}

\section{REFERENCES}

I. Rodbard S. Blood velocity and endocarditis. Circ 1963;27:18-28. https://doi.org// 0.1 161/01.CIR.27.1.18.

2. Kearney RA, Eisen HJ, Wolf JE. Nonvalvular infections of the cardiovascular system. Ann Intern Med 1994;121:219-230. https://doi.org/10.7326/0003-4819-121-3-199408010-00010.

3. Tahara M, Nagai T, Takase $Y$, et al. Primary mural endocarditis without valvular involvement. J Ultrasound Med 2017;36:659-664. https://doi.org// 0.7863/ultra. I 6.03049.

4. Baumgartner H, Falk V, Bax J), et al. 2017 ESC/EACTS Guidelines for the management of valvular heart disease. Joint task force on the management of valvular heart disease of the European Society of Cardiology (ESC): European Association for Cardio-Thoracic Surgery (EACTS). Eur Heart J 2017;38:2739-2791. https:// doi:1 0.1093/eurheartj/eh×391.

5. Mankad R, Herrmann J. Cardiac tumours: echo assessment. Echo Res Pract. 20 16;3(4):R65-R77. https://doi.org/ 10.1530/ERP-16-0035. 